\title{
Cuidados com o recém-nascido após alta hospitalar: orientações aos pais
}

\author{
Care of the newborn after hospital discharge: guidelines for parents \\ Cuidados con el recién nacido tras el alta hospitalaria: orientaciones a los padres
}

Emanoele Busatto

ORCID: https://orcid.org/0000-0002-8205-0540 Universidade Franciscana, Brasil

E-mail: busattoemanoele@outlook.com

Claudia Maria Gabert Diaz

ORCID: https://orcid.org/0000-0003-1100-3242

Universidade Franciscana, Brasil

E-mail: cmgdiaz@bol.com.br

Dielli Arend Teixeira

ORCID: https://orcid.org/0000-0002-9709-6541

Universidade Franciscana, Brasil

E-mail: dielliarend@gmail.com

Pabline Pivetta de Oliveira

ORCID: https://orcid.org/000-0001-5708-9075

Universidade Franciscana, Brasil

E-mail: pablinepivetta@gmail.com

Franceliane Jobim Benedetti

ORCID: https://orcid.org/0000-0002-3334-3910

Universidade Franciscana, Brasil

E-mail: francijb@yahoo.com.br

Regina Gema Santini Costenaro

ORCID: https://orcid.org/0000-0001-8657-2066

Universidade Franciscana, Brasil

E-mail: reginacostenaro@gmail.com

\begin{abstract}
Resumo
A vida contemporânea tem exigido novas metodologias para lidar com a educação e o cuidado com a saúde. Esta pesquisa objetivou descrever os cuidados necessários com o recém-nascido após alta da unidade de terapia intensiva (UTI) e discutir com os profissionais de saúde que atuam em UTI Neonatal sobre as orientações fornecidas aos pais e familiares, bem como conversar com estes sobre o tema. Foi realizada uma pesquisa-ação em um hospital de uma cidade no interior do Rio Grande do Sul, da qual participaram 14 profissionais de saúde e 8 pais e familiares. A partir das respostas ao questionário distribuído aos profissionais, emergiram duas categorias: "Cuidados gerais com a saúde do RN após alta da UTI" e "Cuidados com a alimentação da mãe que está amamentando". Da primeira categoria, surgiram quatro subcategorias: "Cuidados com o ambiente na casa em que o RN vai morar"; "Cuidados com a alimentação do bebê"; "Cuidados com as vestimentas do bebê" e "Cuidados com a higiene do bebê". Dos depoimentos oriundos das respostas dos pais e familiares, emergiram duas categorias: "Preparo para alta da UTI" e "Avaliação da cartilha e principais dúvidas dos pais". Conclui-se que os pais e familiares necessitam de suporte da equipe de saúde, tanto no atendimento hospitalar, como no ambulatorial ou domiciliar. As orientações transmitidas ajudaram muito no cuidado com o filho em casa, por serem de fácil compreensão, esclarecedoras e de acordo com a realidade que vivenciaram com o filho na UTI. Incluir o resumo.
\end{abstract}

Palavras-chave: Cuidado; Recém-nascido; Alta do paciente; Enfermagem.

\begin{abstract}
Contemporary life has demanded new methodologies to deal with education and health care. This paper aims to describe the necessary care for newborn babies after discharge from intensive care unit (ICU) and to discuss with health professionals who work in a Neonatal ICU the guidelines provided to parents and family members, as well as to talk to the latter about the theme. An action research was carried out in a hospital in a city in the countryside of Rio Grande do Sul State, Brazil, in which 14 health professionals and 8 parents and family members have participated. Two categories emerged from the responses to the questionnaire distributed to the health professionals: "General care for the health of the newborn after discharge from the ICU" and "Care for the feeding of the breastfeeding mother. From the first category, four subcategories emerged: "Care for the environment in the home where the newborn will live"; "Care for the baby's food"; "Care for baby's clothes" and "Care for baby's hygiene". From the responses of parents and family members, two categories emerged: "Preparation for discharge from the ICU" and "Booklet
\end{abstract}


evaluation and main doubts of parents". The conclusion of this research is that parents and family members need to receive support from the health team, both in hospital care, as in outpatient or home care. The guidelines provided helped considerably in the care of the child at home, since they are easy to understand, enlightening and in accordance with the reality they experienced with the child in the ICU.

Keywords: Care; Newborn; Patient discharge; Nursing.

\section{Resumen}

La vida contemporánea exige nuevas metodologías para manejar la educación y el cuidado con la salud. Esta investigación buscó describir los cuidados necesarios con el recién nacido tras el alta de la unidad de terapia intensiva (UTI) y discutir con los profesionales de la salud que actúan en UTI Neonatal sobre las orientaciones dadas a los padres y familiares, como también conversar con ellos sobre el tema. Se llevó a cabo una investigación-acción en un hospital de una ciudad del interior de Rio Grande do Sul, en la que participaron 14 profesionales de la salud y 8 padres y familiares. A partir de las respuestas al cuestionario distribuido a los profesionales, surgieron dos categorías: "Cuidados generales con la salud del RN tras el alta de la UTI" y "Cuidados con la alimentación de la madre que está amamantando". De la primera categoría, surgieron cuatro subcategorías: "Cuidados con el ambiente en la casa donde el RN vivirá"; "Cuidados con la alimentación del bebé”; "Cuidados con las vestimentas del bebé” y "Cuidados con la higiene del bebé”. De las declaraciones obtenidas en las respuestas de padres y familiares, surgieron dos categorías: "Preparación para el alta de la UTI" y "Evaluación de la cartilla y principales dudas de los padres". Se concluye que los padres y familiares necesitan soporte del equipo de salud, tanto durante la atención hospitalaria, como en la ambulatoria o domiciliar. Las orientaciones transmitidas ayudaron mucho a cuidar al hijo en casa, por ser de fácil comprensión, claras y acordes a la realidad que vivieron con el hijo en la UTI.

Palabras clave: Cuidado; Recién nacido; Alta del paciente; Enfermería.

\section{Introdução}

Revendo a história da neonatologia, constata-se que a prática da assistência ao recém-nascido, na área da saúde, surgiu no século XIX como um prolongamento da ação da prática obstétrica, na época não existia hospitais infantis e as taxas de mortalidade eram elevadas, especialmente entre os prematuros. Além disso, nesse período, surgiram os primeiros berçários, o desenvolvimento tecnológico, ocorrido após uma Revolução Industrial e um movimento de proteção materno-infantil europeu para garantir natalidade e reduzir o risco de despovoamento e de perdas de mão de obra. A Neonatologia, porém, teve seu início concreto com o obstetra francês Pierre Budin que estendeu sua preocupação com os recém-nascidos (RNs) além das salas de parto, criando um ambulatório de Puericultura em 1892. Na primeira década do século XX observa-se um aumento da contribuição dos pediatras para a medicina neonatal, especialmente com o estudo sobre alimentação e prematuridade (Braga \& Sena, 2012).

Os avanços na Neonatologia reduziram a taxa de mortalidade e a infecção hospitalar foi controlada com o isolamento do RN, porém ocasionaram a separação entre pais e filhos, prejudicando o vínculo afetivo. De acordo com Júlio Hess (autoridade americana em prematuridade), a enfermagem foi fundamental no início da Neonatologia, pois, os melhores resultados obtidos nos cuidados aos RNs prematuros eram alcançados quando enfermeiros bem treinados estavam à frente do serviço como supervisores (Portal Educação, 2017).

Ao pensar em prematuridade, deve-se considerar que os recém-nascidos prematuros (RNPT) apresentam características singulares que requerem adaptação complexa ao meio extra-uterino, no que se refere aos aspectos biológicos, sociais e psicológicos. Nesta perspectiva, há necessidade de uma assistência resolutiva, que deve ser iniciada no pré-natal e ter continuidade após a alta hospitalar. Assim, a continuidade do cuidado, após a alta hospitalar é fundamental para a qualidade de vida do RN que nasceu prematuro e sua família, entretanto, efetivar essa assistência exige um suporte da rede de cuidados em saúde e apoio social, que possui fragilidades no contexto da atenção à saúde da criança no Brasil (Braga \& Sena, 2012).

Neste contexto, a vigilância epidemiológica, estabelece mecanismos que monitoram sistematicamente o atendimento ao $\mathrm{RN}$ de alto risco durante a hospitalização e após a alta, compondo um panorama do desenvolvimento e crescimento dos RNs. Além disso, permite planejar a assistência e propor melhorias de intervenções na UTI e nos ambulatórios. O enfermeiro 
atua como protagonista das estratégias para efetivar a atenção ao RN prematuro, ao possuir habilidades de gerenciamento do cuidado ao egresso de unidade de terapia intensiva neonatal (UTIN) e ainda desenvolve atividades em conjunto com a equipe viabilizando a adesão da família ao acompanhamento. Nesse contexto, o enfermeiro é reconhecido como facilitador do processo de construção de autonomia da família no cuidado ao RN prematuro, após a alta hospitalar (Braga \& Sena, 2012).

Objetivou-se nesta pesquisa descrever os principais cuidados que devem ser prestados ao recém-nascido prematuro ou não, após a alta da UTI e discutir com os profissionais de saúde, que atuam em UTI Neonatal, sobre a importância das orientações que devem ser fornecidas aos pais/família.

\section{Metodologia}

Pesquisa com abordagem qualitativa, a qual permite o pesquisador interpretar e emitir opiniões sobre o fenômeno em estudo. Na abordagem qualitativa os dados são coletados por meio de entrevistas com questões subjetivas (Pereira et al., 2018). Foi realizada uma pesquisa ação, a qual permite a integração e participação entre as pessoas envolvidas no processo (Carpes, Zamberlan \& Costenaro, 2015). Esta pesquisa seguiu cinco etapas: $1^{\text {a }}$ Etapa: Teorização do problema; $2^{\text {a }}$ Etapa: Justificativa e socialização do problema com a equipe envolvida; $3^{\mathrm{a}}$ Etapa: Delimitação de estratégias de intervenção; $4^{\mathrm{a}}$ Etapa: Elaboração da

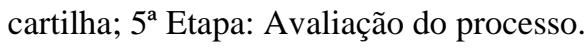

Os cenários desta pesquisa compreenderam uma UTIN e um ambulatório de egresso de um hospital de médio porte de uma cidade do interior do Rio Grande do Sul. Para coleta dos dados, referente ao conteúdo que deveria constar na cartilha, foi distribuído um questionário aos 18 profissionais, dos quais retornaram 14 (enfermeiros, médicos, técnicos de enfermagem) que trabalham na UTI. Também foi realizada uma entrevista, com oito pais/família de RNs egressos da UTIN que estiveram na $1^{\mathrm{a}}$ consulta no ambulatório de egresso. Foram incluídos pais/família, do RN que nasceu prematuro ou não, que estavam na $1^{\mathrm{a}}$ consulta após alta da UTIN e em acompanhamento no ambulatório de egressos; Foram excluídos: Pais/família de RN que residiam fora da cidade de origem da pesquisa; Pais/família de RN que tenha sido transferido para a UTI Pediátrica; Pais/família, cujo filho está na $2^{a}$ ou mais consulta no ambulatório de egressos e pais adolescentes. Os pais serão identificados como pais/família, seguida por um número sequencial na ordem de pesquisa (pais/família 01, pais/família 02...). Esta pesquisa teve registro da CAAE: 80707117.4.0000.5306, e número do parecer: 2.541.965, e assim atendeu a Resolução 466/2012 do CNS/MS, referentes à pesquisa envolvendo seres humanos (Brasil, 2012).

\section{Resultados}

As intervenções seguiram as seguintes etapas: $1^{\text {a }}$ Etapa: Teorização do problema- para iniciar as intervenções foi realizado um encontro com a equipe multiprofissional da UTIN para apresentação da proposta, apreciação e análise da estratégia de elaboração da cartilha com orientações de promoção da saúde. O RN prematuro, após a alta hospitalar, necessita de um acompanhamento rigoroso de seu desenvolvimento e crescimento. O período deste acompanhamento depende das condições vivenciadas no nascimento e no período de internação na UTIN. Por isso, a atenção singular estende-se a família, pois, em muitas vezes, o RN pode necessitar de cuidados especiais após a alta da UTIN. Além disso, a referência e contra referência deve estar atuante e os pais/família, devem contar com os cuidados, orientações e monitoramento dos profissionais de saúde da Estratégia de saúde da família ou Unidade Básica de saúde a que estão vinculados.

$2^{\text {a }}$ Etapa: Justificativa e socialização do problema com a equipe envolvida. No acompanhamento dos RNs no ambulatório de seguimento do RN, percebeu-se uma lacuna no cuidado com o RN, originada pela carência de orientações fornecidas aos pais/família após a alta da UTN.

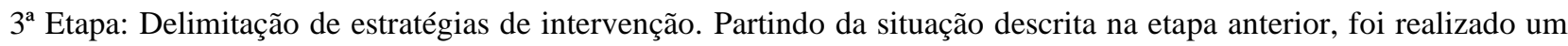


encontro com a equipe multiprofissional da UTIN para discutir e viabilizar a elaboração da cartilha com orientações de promoção da saúde. Assim, os profissionais responderam um questionário sobre os principais cuidados que os pais/família deveriam ter, com seus filhos, após a alta hospitalar da UTI.

$4^{\text {a }}$ Etapa: Elaboração da cartilha- com os itens mencionados pela equipe, nas respostas, oos cuidados foram agrupados de acordo com o tema: Cuidados com a saúde do RN; Cuidados com o ambiente, onde o RN vai ficar; Cuidados com a alimentação do RN; Cuidados com as vestimentas do RN nas diferentes estações; Cuidados necessários com a higiene do RN; Cuidados com a alimentação da mãe que está amamentando. Assim, foi confeccionada a cartilha intitulada "PAIS-FAMÍLIA: Dicas para cuidar do filho recém-nascido", a qual foi distribuída aos pais, no momento da alta do RN, sempre priorizando uma linguagem acessível.

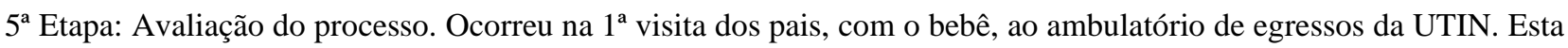
avaliação foi realizada entre o $3^{\circ}$ e $10^{\circ}$ dia após alta hospitalar, período este, significativo, para os pais/família, pois ocorrem os primeiros cuidados com o RN no domicílio. Neste encontro foi realizada uma entrevista com os pais/família, a qual inicialmente abordou dados pessoais, e após foram abordados os cuidados com o filho RN, após a alta hospitalar.

Tendo como questão norteadora: Quais as principais dúvidas que os pais tiveram e se a cartilha auxiliou para esclarecê-las. Os depoimentos oriundos das entrevistas, com os pais, foram analisados por categorização, com base na análise de conteúdo (Bardin, 2016).

A partir das respostas ao questionário emergiram duas categorias: "Cuidados gerais com a saúde do RN após alta da UTI" e "Cuidados com a alimentação da mãe que está amamentando". Salienta-se que na primeira categoria, surgiram quatro subcategorias que são:

Cuidado com ambiente na casa que o RN vai ficar; cuidado com a alimentação do bebê; cuidados com as vestimentas do bebê e cuidados com a higiene do bebê. A seguir serão descritas as categorias, subcategorias e seus conteúdos.

\section{Cuidados gerais com a saúde do bebê após alta da UTI}

Na opinião dos profissionais surgiram os seguintes cuidados:

- $\quad$ As pessoas que seguram o bebê no colo devem estar em boas condições de higiene e devem lavar as mãos;

- $\quad$ Higiene e limpeza do coto do RN; Higiene dos pais e familiares;

- $\quad$ Evitar aglomeração de pessoas nas visitas, limitando a duração do tempo e o número das pessoas nas visitas;

- $\quad$ Evitar o contato do bebê com pessoas tabagistas, resfriadas ou outras infecções;

- $\quad$ Manter consultas e as vacinas em dia;

- Incentivar o aleitamento materno;

- Realizar revisão oftalmológica e teste da orelhinha após a alta.

Com relação ao cuidado com ambiente na casa que o RN vai ficar, foi citado:

- $\quad$ Manter o RN em um ambiente saudável, tranquilo, limpo e arejado;

- Manter sempre boa temperatura para o bebê;

- $\quad$ Evitar contato com familiares fumantes, ou portadores de outras infecções;

- Manter uma temperatura agradável e pouco ruído;

- $\quad$ Colchão deve ser firme;

- Berço com poucos adereços e mantê-lo próximo dos pais. 
Com relação ao cuidado com a alimentação do bebê os profissionais citam:

- $\quad$ Aleitamento materno exclusivo até seis meses, com livre demanda;

- $\quad$ Acordar o bebê para mamar caso esteja muito sonolento;

- Se necessitar de outro tipo de leite seguir orientação médica;

- Se necessário oferecer fórmula, manter rigorosa higiene no preparo, ferver a água e a mamadeira.

Com relação às vestimentas do bebê, nas quatro estações, os profissionais citam:

- $\quad$ Em dias quentes usar roupas leves, com tecido macio, sem muitos adereços;

- $\quad$ Em dias frios usar roupas mais quentinhas (casaquinho e meias), mantendo o conforto do RN;

- Vestir de acordo com o clima evitando excessos ou falta de agasalho a fim de manter a temperatura do RN normal;

- $\quad$ As roupas em geral devem ser confortáveis permitindo que o bebê se movimente livremente;

- Os pais devem observar o excesso de roupas, muita coerência e bom senso.

Nos cuidados com a higiene do bebê, os profissionais citaram que:

- $\quad$ Os pais/família devem manter o banho diário e troca de fraldas sempre que necessário;

- Controlar a temperatura da água, para o banho, que deve estar morna;

- Trocar as fraldas sempre que necessário, removendo resíduos de urina e fezes;

- Usar pomadas conforme necessidade e orientação de saúde, evitando assaduras;

- $\quad$ Sabonete de preferência líquido e com Ph neutro cuidando da pele do RN;

- $\quad$ Se o RN ainda tiver coto umbilical, fazer higiene do mesmo com álcool;

- Se molhar o coto com água, secá-lo bem.

\section{Cuidados com a alimentação da mãe que está amamentando}

Os profissionais salientaram os cuidados com a alimentação da mãe que está amamentando.

- $\quad$ A mãe deve ingerir líquidos em grande quantidade (dois a três litros por dia);

- $\quad$ Manter uma alimentação balanceada, sem dietas restritivas;

- $\quad$ Evitar bebidas alcoólicas e o uso de drogas e de cigarro.

Após esta etapa da análise dos resultados obtidos dos questionários dos profissionais, foi elaborada uma cartilha.

Esta cartilha foi distribuída para os pais/família no momento das orientações para a alta do filho da UTI. Foi solicitado aos pais que lessem com atenção as orientações para que no primeiro dia de retorno no ambulatório de egressos do prematuro, pudessem avaliar se as orientações foram suficientes.

$\mathrm{Na}$ ocasião do retorno ao ambulatório de egressos do prematuro, foi realizada entrevista com os pais, para avaliar a utilização da cartilha. A partir destas entrevistas, emergiram duas categorias, quais sejam: "O Preparo para a alta da UTI"; "Avaliação da cartilha e as principais dúvidas dos pais".

\section{O Preparo para a alta da UTI}

$\mathrm{Na}$ avaliação da cartilha participaram oito pais/famílias. Destes, seis não receberam informações, durante a gestação, sobre a possibilidade de o filho nascer prematuro e duas receberam, sendo que uma gestação eram gemelares e a outra a gestante apresentava hipotireoidismo.

Ao serem questionados sobre o entendimento que tiveram das explicações que os profissionais de saúde forneceram sobre seu bebê durante a internação na UTI, 100\% dos pais/família afirmaram que as orientações eram de fácil compreensão. 
Conforme o depoimento:

Não é necessário mudar nada das orientações da cartilha, pois foram sempre de acordo com as condições do nosso filho, isso é o mais importante os pais terem as informações corretas da situação do seu filho. (Pais/família, 02)

Sempre nos falaram a verdade, e as informações bem compreendidas, com palavras fáceis de entender. (Pais/família, 04)

Os pais/famílias ao relatarem sobre os primeiros cuidados realizados em casa após a alta manifestaram um misto de tranquilidade e medo:

Cuidados foram intensivos, atentos para a respiração do bebê, contando fraldas e eliminações fisiológicas do bebê, preocupações com o manuseio sempre após a higienização correta das mãos e o aleitamento materno exclusivo. (Pais/família, 02)

Um pouco de medo de machucar, mas depois foi tranquilo. (Pais/família, 03)

Seguimos os cuidados conforme aprendemos com o dia a dia delas na UTI neo e conforme orientações do pediatra. A primeira semana foi mais difícil, mas aos poucos tudo vai se ajustando e melhorando conforme conhecemos o bebe. (Pais/família, 04)

Foi bem tranquilo, um alívio de estar bem e em casa. (Pais/família, 05)

Foi tranquilo, tive ajuda do meu marido e da minha mãe, chorava muito(bebê). (Pais/família, 06)

Na primeira semana tivemos insegurança para lidar com nosso bebe, depois com o passar dos dias nos sentimos mais seguros e quando tínhamos dúvidas entramos em contato com os profissionais que cuidaram dela na UTI neo. (Pais/família, 08)

\section{Avaliação da cartilha e as principais dúvidas dos pais}

Com relação às principais dúvidas que os pais/família tiveram para cuidar de seu filho, quatro pais relataram alguns questionamentos: Com relação a amamentação, se deixava o filho só no seio, quando devia tirar o bebê do seio? O medo de dar banho, pois o bebê era muito pequeno? A doença do pulmão pode voltar? Se deveria continuar fazendo fisioterapia em casa? Sobre o cuidado com o contato com outras pessoas?

Os demais pais não tiveram dúvidas, pois foram bem orientados, conforme alguns depoimentos.

Acho que não ficaram dúvidas, pois fomos bem orientados e também tínhamos contato direto com o pediatra se necessário. (Pais/família, 04)

As orientações ajudaram e tinha minha mãe perto e meu marido que sempre ajudou também. (Pais/família, 07)

Com relação a avaliação das orientações descritas na cartilha, 100\% dos pais/famílias afirmaram que ajudaram muito no cuidado com o filho em casa, por serem de fácil compreensão, esclarecedora e de acordo com a realidade que vivenciaram com o filho prematuro. 
No final da entrevista foi solicitado aos pais que deixassem uma mensagem sobre como cuidar de seu filho prematuro quando forem para casa pela primeira vez:

Gostaria de levar uma cartilha a qual pudesse oferecer para as visitas, como cuidados de higiene que a pessoa não deve pegar ou tocar no recém-nascido sem aprovação dos pais. (Pais/famílias, 02)

Que mesmo que pareça difícil o início, tudo se encaminha com força de vontade e persistência, e logo crescem, pois passa muito rápido. A alegria que eles nos proporcionam vale muito a pena. Que os pais sigam os primeiros cuidados com os prematuros conforme foram orientados quanto às visitas e passeios, pois este início é primordial para seu desenvolvimento com saúde, evitando possíveis enfermidades. (Pais/famílias, 04)

Que os pais devem ter paciência, pois cada bebe tem seu tempo; Seguir a cartilha, pois os cuidados são para o benefício do RN. (Pais/família, 01)

Que tenham cuidado maior com eles, prestem atenção em todas as dicas e que fiquem principalmente calmos, cuidar com jeito e muito amor. (Pais/família, 08)

Os pais devem ficar tranquilos, ter calma. Que tenham cuidado com a higiene das mãos para que o pequeno não fique doente de novo. Que deem muito carinho e amor para esses pequenos. (Pais/família 06)

\section{Discussão}

Há uma preocupação dos pais/família e dos profissionais da saúde com a falta de padronização das orientações oferecidas, e que tal problema poderia ser solucionado com um material para direcionar as orientações, didático instrucional escrito que podem modificar a prática de educação em saúde e auxiliar os pais/família na compreensão de informações importantes. Trata-se de uma ferramenta necessária para as orientações, e eficaz quando associado à participação familiar no cuidado do bebê hospitalizado. O material do tipo cartilha é apontado como eficaz para se trabalhar com os pais/família dos bebês internados em unidades neonatais, sendo uma grande vantagem sua disponibilização no domicílio para ser consultado, quando necessário (Santos et al., 2019).

A alta hospitalar planejada, seguida de plano de cuidados, faz parte de um processo complexo que deve envolver, entre outras, a equipe de enfermagem, que se caracteriza como assistência vigilante, humanizada e individualizada. No entanto, observa-se que alguns profissionais ainda não visualizam a atividade como algo essencial na promoção da saúde do prematuro (Silva et al., 2015).

Para que os cuidados com a alta sejam menos dolorosos para os pais, é importante que estes entrem em contato com seu filho o mais breve possível durante a internação. A simples permanência dos pais na unidade neonatal, bem como o estímulo ao toque e conversas com o filho prematuro favorecem o vínculo afetivo profundo e duradouro. Esta vinculação desenvolve efeitos positivos no crescimento e desenvolvimento da criança. Dessa forma, o aconchego dos bebês pelos pais, mesmo quando se encontram em incubadoras ou suportes tecnológicos, deve ser estimulado. Esse contato promove aumento da temperatura corporal do bebê, o que favorece o ganho ponderal e a recuperação da saúde do prematuro (Araújo, Rodrigues \& Pacheco, 2015).

Outro fator importante é esclarecer para a família a respeito do posicionamento adequado no momento do sono do recém-nascido. Nessa perspectiva, os profissionais de saúde têm o dever de orientar esses pais e familiares para o posicionamento de modo correto do RN, mantendo-o em decúbito lateral ou decúbito dorsal (Cesar et al., 2019). 
Com o desenvolvimento dos cuidados intensivos oferecidos aos prematuros, a sobrevida é cada vez maior para bebês cada vez menores. Após dias e até meses de internação chega o momento tão esperado de levar o pequeno ser para casa. Quando a equipe de saúde anuncia a alta, o que deveria ser um sonho se realizando, acaba se tornando fonte de inseguranças, onde se misturam os sentimentos de alegria, medo e fantasias. Com isso surgem muitos questionamentos (Nieto et al., 2016).

O planejamento da alta hospitalar deve ser estratégico e individualizado, considerando as especificidades clínicas do bebê e as condições biopsicossociais da família. O preparo adequado dos pais durante o período de hospitalização, melhorando suas habilidades para os cuidados gerais e específicos ao bebê, além de torná-los mais confiantes para a alta hospitalar, propicia a continuidade dos cuidados no domicílio, aumenta o índice de acompanhamento ambulatorial após a alta e diminui, inclusive, a frequência de re-internações desnecessárias (Bugs et al., 2018).

Mães que conseguiram amamentar de forma exclusiva seu filho em casa atrelam tal fato ao apoio e suporte prévio dos profissionais de saúde, oferecendo ajuda prática quando necessário, assim como incentivos no contato precoce entre mãe e filho e na participação materna no cuidado à mesma ainda no hospital promovem o apego precoce, com desdobramentos para $\mathrm{a}$ manutenção do aleitamento após a alta (Machineski et al., 2018).

Encorajar os pais/família na realização de cuidados, estimularem sua independência e prestar auxílio às mães durante a amamentação atenua o papel materno e paterno, perdido temporariamente, em decorrência da fragilidade do prematuro e de seu internamento na UTIN (Lima et al., 2017).

O desenvolvimento de um material didático instrucional escrito pode modificar a prática de educação em saúde e auxiliar os pais/família na compreensão de informações importantes, sendo apontado como ferramenta necessária para as orientações e é comprovadamente eficaz quando associado à participação familiar no cuidado do filho internado em UTI neonatal, assim a sua disponibilização no domicílio torna-se uma grande vantagem, pois pode ser consultado quando necessário (Santos et al., 2019). Além disso, após a alta do filho da UTI, é normal os pais e a família sentirem-se cansados e preocupados, razão pela qual, se fazem necessárias as redes de apoio que possam atender às suas necessidades (Kurth, et al.,2016).

\section{Considerações Finais}

Vivenciar um parto prematuro traz novas exigências, muitas vezes desconhecidas para o enfrentamento da família à nova situação. Recursos afetivos precisam ser fortalecidos, competências precisam ser aprendidas, habilidades necessitam ser modeladas. Os pais/família precisam do suporte das equipes em todas as esferas de atendimento, seja hospitalar, seja ambulatorial e mesmo domiciliar. As orientações transmitidas ajudaram muito no cuidado com o filho em casa, por serem de fácil compreensão, esclarecedoras e de acordo com a realidade que vivenciaram com o filho na UTI.

Sendo assim, haverá melhor adaptação às solicitações próprias do período de hospitalização, da permanência da mãe na unidade neonatal, aos contatos e às conversas frequentes com a equipe, à compreensão de novas orientações no cuidado do filho. Os resultados desta pesquisa também sinalizam a importância das orientações aos pais, e que estas devem ser realizadas durante todo o período de internação, uma vez que no momento da alta a ansiedade dos pais parece estar voltada para a volta ao lar.

Recomenda-se que mais pesquisas sejam realizadas, com pais de diferentes níveis socioeconômicos e independente de ser o primeiro, o segundo ou o terceiro filho. Isso porque, muitas vezes é posto que os pais com mais filhos, ou que possuem uma situação socioeconômica estável, já possuem conhecimento suficiente, fato este que ainda carece de evidências científicas. 
Research, Society and Development, v. 10, n. 2, e30610212541, 2021

(CC BY 4.0) | ISSN 2525-3409 | DOI: http://dx.doi.org/10.33448/rsd-v10i2.12541

\section{Referências}

Araújo, B. B. M., Rodrigues, B. M. R. D., \& Pacheco, S. T. A. (2015). A promoção do cuidado materno ao neonato prematuro: a perspectiva da educação problematizadora em saúde. Rev Enferm UERJ, 23(1):128-31. http://dx.doi.org/10.12957/reuerj.2015.14779

Bardin L. Análise de Conteúdo. (70a ed.), 2016.

Braga, P. P. \& Sena, R. R. (2012) Estratégias para efetivar a continuidade do cuidado pós-alta ao prematuro: revisão integrativa. Acta Paul Enferm, 25(6):97580 .

Brasil. Ministério da Saúde (2012). Conselho Nacional de Saúde. Resolução no 466, de 12 de dezembro de 2012.

Bugs, B. M., Viera, C. S., Rodrigues, R. M., Conterno, S. F. R., \& Santos, N. T. (2018). Atividade educativa para mães de bebês prematuros como suporte para o cuidado. Rev Enferm Cent-Oeste Min, 8:e2725.

Carpes, A. D., Zamberlan, C., \& Costenaro, R. G. S. (2015). Pesquisa Ação Em Saúde Associada a Outros Dispositivos e Ferramentas. In: Lacerda, MR, Costenaro, RGS. (org. Metodologias da pesquisa para a enfermagem e saúde: da teoria a pratica). 1:110-137.

Cesar, J. A., Marmitt, L. P., Carpena, M. X., Pereira, F. G., Neto, J., Neumann, N. A., \& Acevedo, J. D. (2019). Maternal Knowledge and Unsafe Baby Sleep Position: A Cross-Sectional Survey in Southern Brazil. Maternal and child health journal, 23(2), 183-190. https://doi.org/10.1007/s10995-018-2613-Z

Kurth, E., Krähenbühl, K., Eicher, M., Rodmann, S., Fölmli, L., Conzelmann, C., \& Zemp, E. (2016). Safe start at home: what parents of newborns need after early discharge from hospital - a focus group study. BMC health services research, 16:82. https://doi.org/10.1186/s12913-016-1300-2

Lima, V. F., Mazza, V. A., Mór, L. M., \& Pinto, M. N. G. R. (2017). Vivência dos familiares de prematuros internados em unidade de terapia intensiva neonatal. REME - Rev Min Enferm, 21:e-1026. http://www.dx.doi.org/10.5935/1415-2762.20170036.

Machineski, G. G., Reis, N. N., Vieira, C. S., Toso, B. R. G. O., \& Caldeira, S. (2018). Percepção das mães quanto à competência materna nos cuidados domiciliares do recém-nascido prematuro. Rev Saúde - Sta. Maria, 44 (3):1-14. 10.5902/2236583431627.

Nieto, G. C. S., Rugolo, L. M., Sadeck, L., Silveira, R. C., \& Garbers, R. (2016). Nascer prematuro: manual de orientação aos pais, familiares e cuidadores de prematuros na alta hospitalar. 1 ed. Rio de Janeiro: Elsevier, 64p.

Pereira, A. S., Shitsuka, D. M., Parreira, F. J., \& Shitsuka, R. (2018). Metodologia da pesquisa científica. UFSM.

Portal da Educação. Histórico da neonatologia. Campo Grande, MS. Publicado em 27 jul. 2017.

Santos, A. S., Rodrigues, L. N., Silva, W. C. P., Brito, L. L. M. S., Viana, M. C. A, \& Chaves, E. M. C. (2019) Educação em saúde na unidade de terapia intensiva neonatal. Rev Enferm Atual In Derme 89(27). https://doi.org/10.31011/reaid-2019-v.89-n.27-art.35.

Silva, C. M. S., Dantas, J. M., Souza, F. M. L. C., Silva, R. A. R., Lopes, T. R. G., \& Carvalho, J. B. L. (2015). Sentimentos vivenciados por puérperas na realização do primeiro banho do recém nascido no alojamento conjunto. O Mundo da Saúde, São Paulo. 39(3):279-286. 10.15343/01047809.20153903279286 